2009-01-01

\title{
Raman spectroscopy: a potential platform for the rapid measurement of carbon nanotube-induced cytotoxicity
}

\author{
Peter Knief \\ Technological University Dublin \\ Colin Clarke \\ Technological University Dublin, Colin.Clarke@tudublin.ie \\ Eva Herzog \\ Technological University Dublin, eva.herzog@tudublin.ie
}

See next page for additional authors

Follow this and additional works at: https://arrow.tudublin.ie/radart

Part of the Pharmacology, Toxicology and Environmental Health Commons

\section{Recommended Citation \\ Knief, Peter et al (2009) Raman spectroscopy: a potential platform for the rapid measurement of carbon nanotube-induced cytotoxicity. Analyst, Vol. 134, no. 6, pp.1182-1191. doi:10.1039/b821393c}

This Article is brought to you for free and open access by the Radiation and Environmental Science Centre at ARROW@TU Dublin. It has been accepted for inclusion in Articles by an authorized administrator of ARROW@TU Dublin. For more information, please contact arrow.admin@tudublin.ie, aisling.coyne@tudublin.ie, gerard.connolly@tudublin.ie.

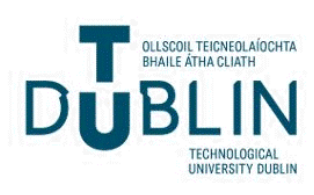


Authors

Peter Knief, Colin Clarke, Eva Herzog, Maria Davoren, Fiona Lyng, Aidan Meade, and Hugh Byrne 


\title{
Raman spectroscopy - a potential platform for the rapid measurement of carbon nanotube-induced cytotoxicity
}

\author{
Peter Knief*', Colin Clarke², Eva Herzog ${ }^{2}$, Maria Davoren ${ }^{2}$, Fiona M. Lyng ${ }^{2}$, Aidan D. Meade ${ }^{1}$, and Hugh \\ J. Byrne ${ }^{3}$
}

\author{
${ }_{5}$ Received (in $\left.X X X, X X X\right) X t h X X X X X X X X X 200 X$, Accepted Xth $X X X X X X X X X 200 X$ \\ First published on the web $X$ th $X X X X X X X X X 200 X$ \\ DOI: 10.1039/b000000x
}

In this study the suitability of Raman spectroscopy for the determination of carbon nanotube mediated toxicity on human alveolar carcinoma epithelial cells (A549) is explored. The exposure 10 of this cell line represents the primary pathway of exposure in humans, that of inhalation. Peak ratio analysis demonstrates a dose dependent response which correlates to previous toxicological studies. Principal component analysis is employed to further classify cellular response as a function of dose and to examine differences between spectra as a function of exposed concentration. To further illustrate the potential of Raman spectroscopy in this field, Partial Least ${ }_{15}$ Squares (PLS) regression and genetic algorithm feature selection have been utilised to demonstrate that clonogenic end points, and therefore toxic response, can be potentially predicted from spectra of cells exposed to un-determined doses, removing the need for costly and time consuming biochemical assays. This preliminary study demonstrates the potential of Raman spectroscopy as a probe of cytotoxicity to nanoparticle exposure.

\footnotetext{
* Author to whom correspondence should be addressed.

${ }^{1}$ School of Physics/Focas Research Institute, Dublin Institute of Technology, Kevin Street, Dublin

${ }^{2}$ Radiation and Environmental Science Centre, Focas Research Institute, Dublin Institute of Technology, Kevin Street, Dublin 8

${ }^{3}$ Focas Research Institute, Dublin Institute of Technology, Kevin Street, Dublin 8
} 


\section{Introduction}

Nanomaterials are considered to be a new class of materials with unusual characteristics, not only due to the chemistry of the materials themselves, but because their dimensions result in new 5 physical characteristics that have a significant impact on their chemical properties ${ }^{1}$. In addition, chemical modifications can change the optical, magnetic or electric properties of these materials. Thus nanomaterials are a new class of materials that can be employed in wide ranging applications in nano- science, 10 medicine and engineering.

Carbon nanotubes, in both their multi- and single-walled forms, have attracted significant attention since their emergence in $1991^{2}$. They are one dimensional macro molecules of rolled graphene sheets, either single or multi walled, with diameter of 15 the order of nanometers and a length up to several centimetres. It is anticipated that their huge potential will see them in mass production in the near future ${ }^{3}$. Given the likely widespread applications of these materials, concerns exist regarding potential toxic responses and an evaluation of their biological effects is 20 required. Various studies have already been published reporting toxicological or inflammatory responses in animal models ${ }^{4-6}$. On a cellular level, some in vitro studies have shown less dramatic effects in terms of viability and proliferative capacity ${ }^{7}$ although chemical modification or functionalisation of the nanotubes can 25 increase the toxic response ${ }^{8}$. The diversity of the reports of the extent and mechanism of the toxic response however clearly demonstrates the need for more systematic investigations.

Recent studies have shown that common colorimetric assays interact with single walled carbon nanotubes (SWCNT) 30 themselves, compromising their overall suitability in toxicological assays ${ }^{9,10}$. The cell culture medium itself is altered by the interaction with $\mathrm{SWCNTs}^{11}$, challenging conventional cytology in general. Clonogenic studies have been demonstrated to be a potentially more appropriate toxicological assay and it has 35 been demonstrated that colony-size rather than colony number is a more powerful measurement of cellular toxicity post exposure to SWCNTs $^{12}$. The implications are that the toxic response is possibly one of reduced proliferative capacity of the cells due to medium depletion rather than a reduced viability due to direct 40 interaction with the cells ${ }^{12}$. Such an indirect toxicological response has more recently been demonstrated ${ }^{13}$. While the clonogenic assay yields a reliable assessment of the toxic response to SWCNT exposure in vitro, the endpoints of colony size and number are time consuming, phenomenological and

45 limited in their potential to elucidate underlying biochemical mechanisms. Therefore alternative assaying techniques are desirable for the rapid evaluation of cellular toxicity post exposure to carbon nanotubes.

Raman spectroscopy is a very versatile analytical tool, known 50 for its strengths in the physical and chemical characterisation of materials and systems. This technique has previously been employed for the analysis of interactions of SWCNT's with biomolecules as well as the effect of external toxins as agents for induced cell damage ${ }^{14}, 15$. The modality potentially offers
55 analytical and diagnostic information at a high sub-cellular spatial resolution. It derives additional benefit from the minimal need for processing of biological materials. It has already been shown to be a viable tool for disease diagnosis ${ }^{16}$ as well as for the detection of alterations on a cellular level caused by external influences ${ }^{17}$.

${ }_{60}$ The Raman spectrum of a cell also contains chemical information regarding its constituents, providing a complete biochemical fingerprint of the cell, and ultimately exhibiting signatures that are indicative of cell state, e.g. proliferating, apoptotic, necrotic, etc. ${ }^{18}$. While changes to individual or combinations of spectral ${ }_{65}$ features may give clear indications of cellular response, the complex biochemical changes are often manifest as multivariate changes to the overall the spectral response. Multivariate analysis of spectroscopic data delivers an appropriate means to analyse multiple dependent and independent singular features occurring 70 in vibrational spectra of biological materials, delivering a detailed view of the overall response ${ }^{19,20}$, allowing classification of, for example, tissue pathologies and quantification of response to external stimuli with the additional option of modelling the spectral features for prediction of a biological result.

75 In this study, Raman spectroscopy is employed as a probe of the toxic response of cellular systems to SWCNT exposure in vitro. A human lung cell line was chosen for experimental purposes, as it represents the potential scenario of inhalation, one of the first steps in the route of exposure. In order to allow a 80 realistic relationship between the experimental data presented here and that in vivo, the SWCNT samples were minimally processed to mimic inhalation of airborne SWCNT dust particles. SWCNT dispersion was carried out in an identical fashion to that employed in previous cytotoxicity studies ${ }^{9-11}$ and cell growth and 85 exposure conditions were identical to those employed in previous clonogenic studies $^{12}$. Dose dependent responses are examined in terms of peak ratios utilised in previous toxicity studies as well as principal component analysis. Finally, the suitability of genetic algorithm optimised Partial Least Squares (PLS) regression as a 90 quantitative model to predict clonogenic endpoints is assessed. Coupled with such a predictive model, spectroscopic analysis is demonstrated to be a potentially powerful analytical technique avoiding time consuming and expensive biochemical assays.

\section{Experimental Procedures}

\section{${ }_{95}$ Cell culture}

Quartz slides (24.5mm x 24.5mm, UQG Optics Ltd.) were coated for $24 \mathrm{~h}$ at $4{ }^{\circ} \mathrm{C}$ with a sterile solution of $2 \%$ gelatine (Type-B from bovine skin) in deionised water $\left(\mathrm{dH}_{2} \mathrm{O}\right)$ solution. Such substrates have previously been shown to be optimal for cell 100 growth and subsequent spectral analysis ${ }^{17}$. Cells of the human alveolar carcinoma epithelial line A549 (ATCC, CCL-185), were cultivated in Dulbecco's modified minimum essential medium (DMEM, Cambrex). All media were supplemented with $10 \%$ foetal bovine serum (FBS) and $45 \mathrm{IU} \mathrm{ml}^{-1}$ penicillin and $45 \mu \mathrm{g}$ $105 \mathrm{ml}^{-1}$ streptomycin and cells were maintained at $37^{\circ} \mathrm{C}$ in a $5 \% \mathrm{CO}_{2}$ humidified incubator. The cells were allowed to attach to the quartz substrates at a concentration of approximately $2 \times 10^{6}$ cells per slide for $24 \mathrm{~h}$. After the $24 \mathrm{~h}$ incubation period, the unattached cells were rinsed off with PBS. An ultrasonic tip (Ultra sonic 
processor VCX-750 watt) operating at $40 \%$ was employed to disperse the SWCNTs in four exposure suspensions of single wall carbon nanotubes $(0 \mathrm{mg} / \mathrm{l}$ (Control), $1.56 \mathrm{mg} / \mathrm{l}, 6.25 \mathrm{mg} / \mathrm{l}, 25.0$ $\mathrm{mg} / \mathrm{l}, \quad 100 \mathrm{mg} / \mathrm{l})$ in supplemented medium. HiPco Carbon ${ }_{5}$ Nanotubes (Carbon Nanotubes Inc.) were employed for the study for consistency with previous studies ${ }^{9-12}$. The tip was operated at a medium level of output for a total time of 30 s carried out in $10 \mathrm{~s}$ sequential steps to minimise sample heating. The cells were then exposed to $3 \mathrm{ml}$ of each of the different SWCNT suspensions for 1096 hours. After the exposure period the slides were rinsed with PBS and fixed in 4\% formalin in PBS solution for 10 minutes, rinsed once again in $\mathrm{dH}_{2} \mathrm{O}$, and finally stored in $\mathrm{dH}_{2} \mathrm{O}$ at $4{ }^{\circ} \mathrm{C}$ prior to conducting the measurements. Three independent sets of cells were exposed at each concentration and all measurements 15 were repeated for each exposure batch.

\section{Spectroscopy}

Raman Spectroscopy was carried out with an Instruments S.A. (Horiba Jobin-Yvon) Labram 1B Raman confocal microscope using $514.5 \mathrm{~nm}$ laser excitation with a grating of $1800 \mathrm{l} / \mathrm{mm}$, 20 providing a spectral dispersion of about $1 \mathrm{~cm}^{-1}$ per pixel. Spectra were recorded using a water immersion lens (Olympus Lum-Plan FL 100x) from substrates immersed in water in a climatic chamber to prevent desiccation of the samples. The immersion reservoir was constructed by inserting a quartz window into the 25 bottom of a Petri dish filled with $\mathrm{dH}_{2} \mathrm{O}$. The $\mathrm{x} 100$ water immersion objective gave a spot size approximately $1 \mu \mathrm{m}$ at the sample.

All recordings were performed as an average of three individual measurements of one point to reduce the influence of 30 spectral noise. The system was previously calibrated to the spectral line of crystalline silicon, at $520.7 \mathrm{~cm}^{-1}$ at a constant room temperature of $21^{\circ} \mathrm{C}$. The measurement range was set to an interval of $\sim 250-1750 \mathrm{~cm}^{-1}$ in order to detect spectra within the fingerprint region of the cell samples ${ }^{17}$ and the characteristic ${ }_{35}$ SWCNT features ${ }^{14}$. Before spectral acquisition, the dark current of the system and the system intensity response (using the NIST fluorescent intensity standard SRM 2243), were recorded in triplicate. After a series of spectral measurements on a particular slide, the spectral background of the substrate was acquired. The 40 laser power was set to $23 \mathrm{~mW}$ at the sample and the acquisition time was set to 90 s which delivered reasonable spectra.

In total, approximately 75 spectra ( 25 per sample in triplicate) were recorded from the nuclear portion of multiple cells at each concentration. Principal component analysis (PCA- see 45 following section for details) was employed to identify outlier spectra $^{21}$. Cells across the whole area of the sample slide were chosen for measurement in an attempt to ensure a true representation of the sample. This technique was designed to limit variability that might occur due to the spatial position of the 50 laser focal spot within the nuclear portion of cells, and biological variability that could occur between samples of the cell line. It was noted however, that even after repeated washing with PBS, some single wall carbon nanotube aggregates could be visibly observed attached to the cells, although no SWCNTs were 55 observed inside the cells themselves ${ }^{9}$. All measurements reported here were taken away from regions where large aggregates were visible.

\section{${ }_{60}$ Data analysis}

In total 321 valid spectra were acquired for the 5 distinct concentrations (Table 1) with a spectral range from 248 to 1751 $\mathrm{cm}^{-1}$. The raw spectra were imported into Matlab 7.3 (Mathworks CA, USA) for pre-processing and analysis. Every spectrum was 65 corrected for system intensity response, according to the guidelines of NIST (SRM 2243) ${ }^{22}$. Prior to the subtraction of the underlying quartz signature, each spectrum was normalised to the characteristic underlying quartz peak at $486 \mathrm{~cm}^{-1}{ }^{23}$, without the application of any filtering. Finally the spectra were cropped to a 70 spectral window of $599-1700 \mathrm{~cm}^{-1}$ to isolate the fingerprint region. In order to minimise the electronic noise associated with the CCD detector, the spectra were smoothed using the Savitzky Golay algorithm ${ }^{24}$ with a 15 point window and a polynomial order of 3 for further analysis.

75 Table 1: Sample numbers after recording the measurements and outlier removal

\begin{tabular}{ccccccc} 
Sample & \multicolumn{3}{c}{ Recorded Replicates } & \multicolumn{3}{c}{ Validated Replicates } \\
Concentration & I & II & III & I & II & III \\
$0.0 \mathrm{mg} / 1$ & 25 & 25 & 25 & 22 & 21 & 20 \\
$1.56 \mathrm{mg} / 1$ & 25 & 25 & 25 & 19 & 21 & 23 \\
$6.25 \mathrm{mg} / 1$ & 25 & 25 & 25 & 22 & 20 & 21 \\
$25.0 \mathrm{mg} / 1$ & 25 & 25 & 25 & 22 & 24 & 21 \\
$100.0 \mathrm{mg} / 1$ & 25 & 25 & 25 & 21 & 23 & 21
\end{tabular}

\section{Principal component analysis}

Principal component analysis (PCA) is a method of multivariate 80 statistical analysis broadly used with datasets of multiple dimensions. It allows the reduction of the number of variables in a multidimensional dataset while retaining most of the variation within the dataset. The principal components (PCs) obtained are not correlated to each other and are also called eigenvectors or 85 loadings. The lack of correlation means that the PCs represent valuable different 'dimensions' of the data. The order of the PCs describes their relative importance for the dataset. $\mathrm{PC}_{1}$ describes the highest amount of variation, $\mathrm{PC}_{2}$ the second greatest and so on. The variance of the $\mathrm{PC}$ is sometimes called the eigenvalue or 90 scores of the PC. When PCA is successfully applied, the eigenvalues of the PCs are progressively lower and ideally the variation in the data set can be described sufficiently by a few PCs whose eigenvalues are most significant. The actual number of PCs that feeds into further analysis is dependent on the 95 variance covered by the eigenvalue of a PC, visualized by e.g. a Scree plot, and the threshold one applies, in order to give a satisfactory representation of the original dataset, explaining most of the variance within ${ }^{25,26}$. As the scores of a sample in PCs are orthogonal to each other and uncorrelated they represent 100 coordinates along the dimensions of the PCs in e.g. a three dimensional space for 3PCs, used to access possible separation of certain groups within samples. Analysing the loadings of a PC can give information about the variable based source for the variance in a PC. Variables of the loadings within the PC with 105 positive value indicate a positive contribution to the nature and dimension of the PC whereas negative values show an inverse relation to the $\mathrm{PC}$, not giving a positive contribution to the variance covered by the $\mathrm{PC}$ and therefore not contributing to the dimension of the PC. 
In this study the datasets for all exposure concentrations were pre-processed as described previously, arranged conforming to the required SAISIR structure (SAISIR (2008)(c). Package of function for chemometrics in the MATLAB (Registered) 5 environment. Dominique Bertrand coordinator. Unité de Sensométrie et de Chimiométrie ${ }^{27}$ ) and then fed into PCA analysis using Matlab 7.3 (Mathworks CA, USA) with the accompanying statistics toolbox. In a second PCA analysis the spectra were doubly derivatized, a common technique in dealing 10 with spectral data to further reduce the baselines and backgrounds $^{28}$, and also subjected to PCA using Matlab.

\section{Partial least squares modelling}

First described by Wold in 1960, partial least squares (PLS) is a popular and well known tool in the field of chemometrics ${ }^{29-31}$. 15 The aim of PLS is the construction of a model to describe the response variables (i.e. analyte concentration) in terms of the observed variables (spectra) from a set of training data. The least squares model is given by:

20 $Y=X B+E$

Equation 1

where $Y=n \times m$ are the dependant variables (i.e. concentration), $X=n \times p$ are the independent variables (i.e. Raman spectra), $B=p \times m$ matrix of regression parameters for each component 25 in $\mathrm{Y}$ and $\mathrm{E}$ are the matrix of residuals (differences between measured and predicted variables). PLS decomposition is similar to that of principal component analysis (PCA). PCA produces factors based on variance solely within the $X$ matrix whereas PLS considers both the $X$ and $Y$ matrices ensuring the factors correlate 30 the $X$ matrix to the concentration. PLS differs from similar techniques such as multiple linear regression (MLR) and principal component regression (PCR) in that the $X$ and $Y$ variables are decomposed simultaneously maximizing the covariance between both matrices and allowing direct correlation 35 between the spectra and target concentrations ${ }^{30}$. In addition to the scores and loading matrix, a series of weight vectors are calculated which enhance the variables with high correlation to the targets. The initial weight vector is calculated as follows:

${ }_{40} w_{1}=X^{T} y /\left(\left\|X^{T} y\right\|\right)$

Equation 2

The initial scores vector is calculated as:

$$
t_{1}=X w_{1}
$$

Equation 3

and the loadings:

$$
p_{1}=X^{T} t_{1} /\left(\left\|t_{1}^{T} t_{1}\right\|\right)
$$

Equation 4

50 The regression parameters are calculated as follows:

$$
\hat{b}=y^{T} t_{1} /\left(t_{1}^{T} t_{1}\right)
$$

Equation 5

The residual matrix is calculated as:

55

$$
E_{1}=X-t_{1} p_{1}^{T}
$$

Equation 6

The algorithm continues for each factor used, taking $E_{l}$ instead of the weight matrix to calculate the second set of weights. When
60 presented with an unknown spectrum, $y$ is determined using $W$ and $P$ to compute scores for the unknown spectrum along with the regression parameters allowing the concentration of $y$ to be determined from equation 1. PLS calibration models were constructed in this work using the SIMPLS algorithm with root 65 mean squared error of cross validation (RMSECV) as the fitness value. Leave one out cross validation was used to select the number of latent variables (LVs) to retain. To construct the PLSmodels the Eigenvector toolbox 3.5 for MATLAB was used.

\section{Feature selection using genetic algorithms}

70 Calibration models are known to be greatly improved through the application of efficient feature selection methods, increasing the predictive ability and reducing model complexity. One such method is the adaptive search technique known as the genetic algorithm (GA). Here a GA based variable selection procedure is 75 used to reduce the original spectra to a subset of wavenumbers to correlate Raman spectra to response. The first generation for evaluation is a random population consisting of a number of individuals or "chromosomes", each containing a subset of the original variables. Each chromosome is composed of a vector of $801 \mathrm{~s}$ and $0 \mathrm{~s}$, corresponding to the wavenumbers in the $X$ matrix, ( 1 if selected and 0 if not) where each wave number is termed a "gene". The performance of models resulting from each chromosome is determined by means of a fitness function (here the root mean square error of cross validation is used). Once each 85 generation is evaluated a new set of chromosomes is produced by retaining and "crossing" the fittest individuals from the previous generation. "Mutations" are also produced which force the evaluation of new combinations avoiding saturation with similar sets of events and can further lower the number of variables and 90 fitness values. The process continues until the difference in mean fitness level between successive generations is below a certain threshold the GA is terminated to avoid over-training and avoid over fitting risk in the PLS model ${ }^{32-34}$. Feature selection in this work was achieved using GA optimisation (with the genpls 95 MATLAB toolbox by Ledardi) over 100 runs requiring approximately 60mins (see table 1 for GA settings).

Each calibration model was evaluated using root mean squared error of cross validation (RMSECV) and root mean squared error of calibration (RMSEC) performed on the 100 calibration set. The root mean squared error of prediction (RMSEP) of the independent testing is also calculated. The root mean squared error is calculated for each dataset as follows:

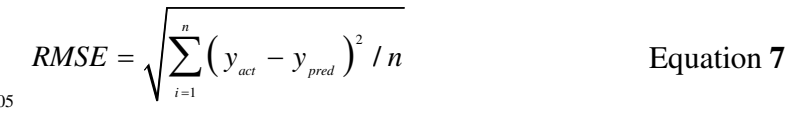

In this study PLS regression enhanced through GA feature selection is used to construct a calibration model to predict the end points of clonogenic studies directly from dose dependant Raman spectra. In order to ensure over fitting is avoided the data 110 is split into two sets, a calibration and testing set. Firstly the optimum number of latent variables is chosen with cross validation and the model constructed using the calibration set. The test set is unseen during the training phases and is used as an independent test set validation of the constructed model.

\section{${ }^{115}$ Results and Discussion}




\section{Preliminary Spectral Evaluation}

The Raman spectrum of a SWCNT sample (suspended in water at concentrations similar to those used during this study) exhibits characteristic radial breathing modes (RBM) in the region of ${ }_{5} 200 \mathrm{~cm}^{-1} 35$ (Figure 1). These features describe the synchronous oscillation of the atoms of the nanotube in the radial direction and can be used to define structural characteristics of SWCNTs such as their diameter, metallicity, and helicity ${ }^{36}$. The so-called "disorder-included" D band appears at $1330-1390 \mathrm{~cm}^{-1}$ and is 10 reputedly an indicator for disorder in the graphene sheet. The tangential mode, or G-Band, appears at $1583-1605 \mathrm{~cm}^{-1}$ originating from tangential oscillations of the carbon atoms in the nanotubes ${ }^{36-38}$.

Spectra of A549 cells Figure 2 (A) show classic features 15 within the amide I band area of 1656-1690 $\mathrm{cm}^{-1}$, consisting of $\sim 80 \%$ of $\mathrm{CO}$ stretching , $\sim 10 \% \mathrm{CN}$ stretching and $\sim 10 \% \mathrm{NH}$ bending vibration modes, indicating protein based $\alpha$-helix, random coil and $\beta$-sheet structures. In the amide III area at about $1238 \mathrm{~cm}^{-1} \beta$-sheet and random coil structures are indicated by $20 \sim 30 \% \mathrm{CN}$ stretching and $\sim 30 \% \mathrm{NH}$ bending vibrations, as well as $\sim 10 \% \mathrm{CO}$ stretching and $\sim 10 \% \mathrm{O}=\mathrm{C}-\mathrm{N}$ bending vibrations. Vibrational features of amino acids and amino acid hydro halides appear in the area of $1485-1660 \mathrm{~cm}^{-1}$ (NH deformation vibrations and $\alpha$-form $\mathrm{C}=\mathrm{O}$ stretching of polypeptides). ${ }_{25}$ Characteristic signals of lipid related groups appear at $965 \mathrm{~cm}^{-1}$ (CN asymmetric stretching vibrations), $1170 \mathrm{~cm}^{-1}$ (weak CO-O-C symmetric stretching) and $1451 \mathrm{~cm}^{-1}\left(\mathrm{CH}_{2}\right.$ scissoring and $\mathrm{CH}_{3}$ bending vibrations $)^{18,39-41}$. As the samples were rinsed before fixing as described in the cell culture section above, it is assumed 30 that all features are cellular in origin. In Figure 2 (B), an average spectrum of a high concentration exposure cellular sample $(25 \mathrm{mg} / \mathrm{l})$ is shown. Strong contributions of the G-Line \& D-Line features of SWCNTs as well as common cellular spectral features are clearly visible, although the SWCNTs were washed off

35 thoroughly and were not visible microscopically. In a previous study, in samples prepared under identical conditions, no SWCNTs could be observed internalised in the cells and so it is assumed that small bundles or ropes adhere to the cell surface? The strongest peak of the typical SWCNT spectrum, the G-line at 40 about $1585 \mathrm{~cm}^{-136}$, overlaps strongly with the amide I region of the cellular spectra $\left(1637,1656-1690 \mathrm{~cm}^{-1}\right)^{39,42}$. This makes it difficult to utilise this band for analysis of cellular response to the SWCNT exposure without deconvolution.

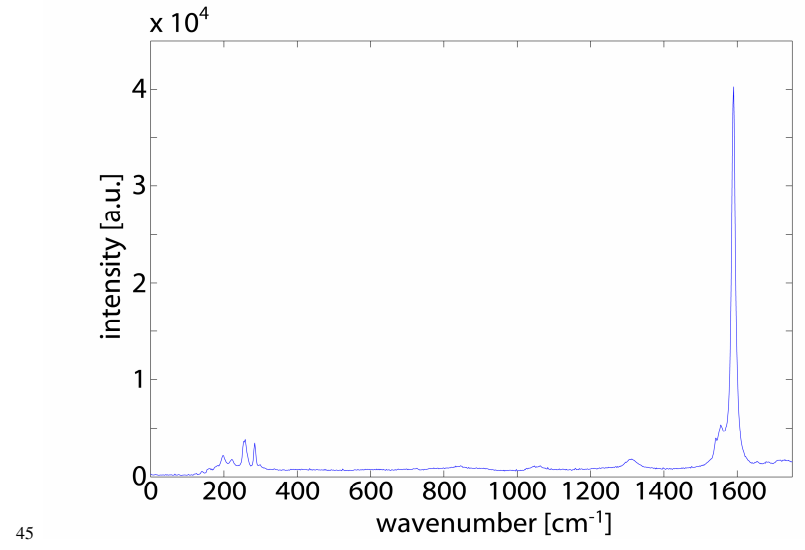

Figure 1. Raman Spectrum of SWCNT with characteristic features (RBM'S at $\sim 180-300 \mathrm{~cm}^{-1}$, D-Line at $\sim 1350 \mathrm{~cm}^{-1}$, G-line at $\sim 1585 \mathrm{~cm}^{-1}$ )

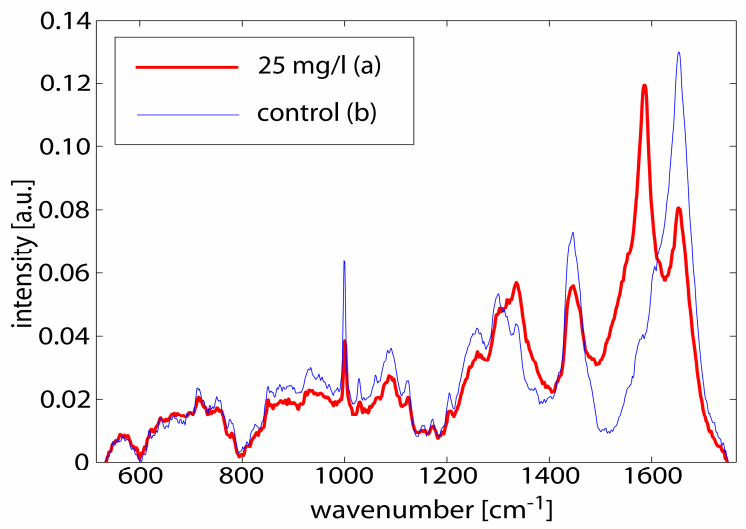

50 Figure 2. Raman Spectra of A549 exposed Cells ( $25 \mathrm{mg} / \mathrm{l} \mathrm{SWCNT)} \mathrm{(a)}$ and A549 control cells (b), smoothed with Savitzky Golay Filter order 3, 15 points.

After background removal, the region of $1502-1700 \mathrm{~cm}^{-1}$, was extracted and fitted with a mixture of Gaussian/Lorenzian band ${ }_{55}$ functions to extract the relative contributions of the SWCNT Gline and the cellular amide I band. Figure 3 shows the intensity of the SWCNT G-line as a function of exposure dose in terms of concentration $(\mathrm{mg} / \mathrm{l})$. Although the Raman intensity is approximately linear as a function of dose up to $\sim 30 \mathrm{mg} / \mathrm{l}$, the 60 maximum dose of $100 \mathrm{mg} / \mathrm{l}$ shows significant deviation from this. This apparent saturation of the response may be a result of over dosage, the nanotubes not being effectively dispersed throughout the sample, and/or an effect of the increased optical density of the residual carbon nanotubes which are resonant at the Raman 65 wavelength, limiting penetration of the light into the sample and absorption of the light scattered by the sample. Figure 4 shows the intensity of the amide I Raman band as a function of SWCNT dosage. The intensity is seen to be only weakly dependent on dosage, indicating that the reduced intensity of the SWCNT G70 line has origin primarily in saturation of dosage rather than optical effects, although the slight reduction at large doses points towards some optical effects. Such a saturation of dosage resulting in large aggregates of nanotubes adhered to the cells has indeed been seen in other studies ${ }^{9}$.

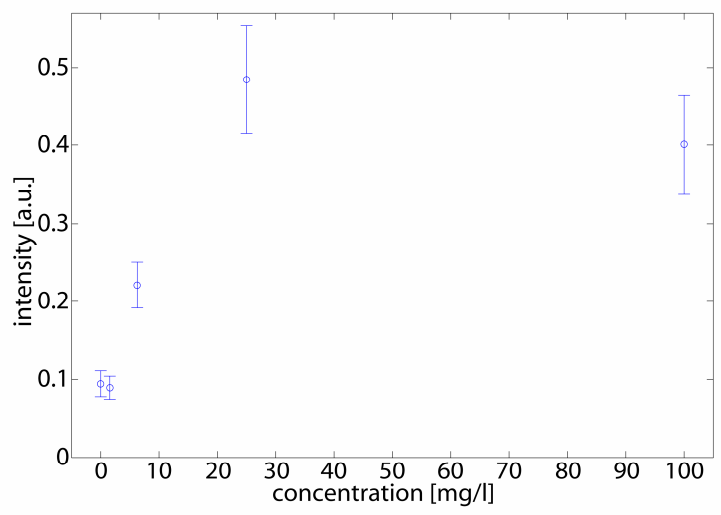

Figure 3. Intensity of G-Line at $\sim 1585 \mathrm{~cm}^{-1}$ versus concentration 


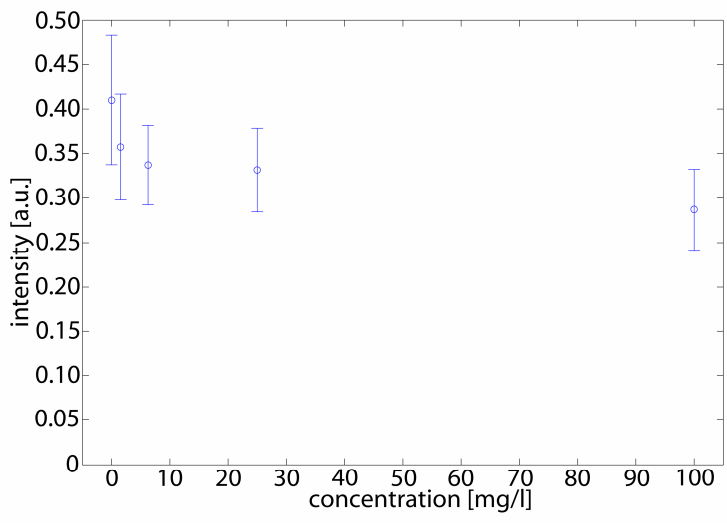

Figure 4 . Intensity of amide I at $1656 \mathrm{~cm}^{-1}$ versus concentration

A direct visual comparison of the cellular Raman spectra demonstrates clearly that several individual peaks are altered as a 5 result of exposure (Figure 5). Examination of the spectra reveals changes to the $1030-1060 \mathrm{~cm}^{-1}$ lipid related bands caused by $\mathrm{C}$ O-P stretching and CO-O-C sym. stretching $\sim$ vibrations ${ }^{18,40,41}$, an observation which correlates well with the recent work of Davoren et al. ${ }^{9}$ which, using Transmission Electron Microscopy 10 (TEM), demonstrated an increase of surfactant storing lamellar bodies in A549 cells after exposure to SWCNTs, which supports the assumption of a change in the overall lipid content in the exposed cells. It should be noted that although the nucleus is primarily targeted, the spot of $\sim 2-3 \mathrm{~mm}$ diameter will pick up 15 some of the neighbouring and overlaying cytoplasm. Changes to the RNA ribose C-O vibration at $930-960 \mathrm{~cm}^{-1}$ and $1295-1304 \mathrm{~cm}^{-}$ ${ }^{1}$, as well as changes to the adenine and guanine activity at $\sim 1345$ $\mathrm{cm}^{-1} 39,42,43$ are also observed. It is possible, however, that the Dline of the SWCNTs may be masking the response in this latter 20 region, but changes to the cellular spectra are clearly visible after deconvolution of the amide III band area, far from the spectral features of the SWCNTs, with its different conformations at approximately $1238,1258,1271 \mathrm{~cm}^{-1}$ by a combined Gaussian and Lorenzian fit, known as a pseudo-Voigt function ${ }^{44,45}$ to

25 approximate the Voigt profile, with a total of nine individual centred peaks, identified from the second derivative of the unfitted region ${ }^{15}$.

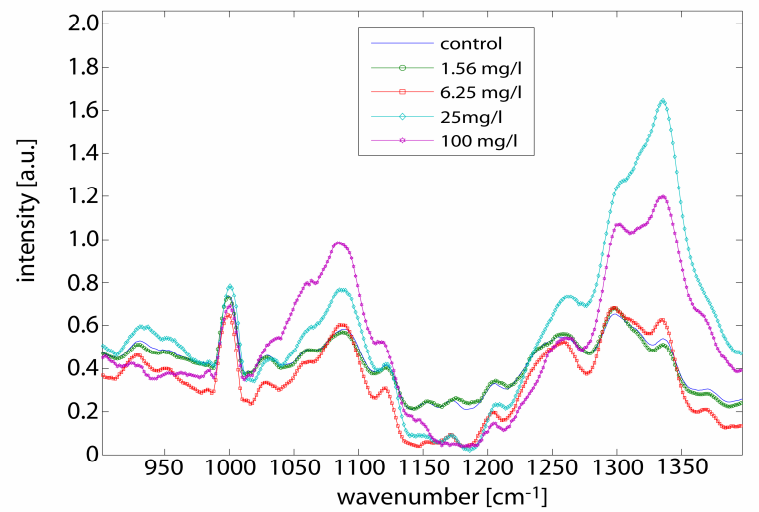

Figure 5. Detailed magnification of Raman Spectra of the A549 Cells at 30 different concentrations smoothed with Savitzky Golay Filter order 3, 15 points.
Although difficult to precisely assign the many overlapping bands, the use of the ratio of Raman peak heights for $\mathrm{CH}_{2}$ deformation modes at $\sim 1302 \mathrm{~cm}^{-1}$, DNA bases guanine, adenine 35 and thymine at 1287 and $1338 \mathrm{~cm}^{-1}$ versus the amide III band at $1238 \mathrm{~cm}^{-1}$ have previously been used for estimation of cellular toxicity ${ }^{15}$. Figure 6, 7 and 8 show the dose dependent response of these peak ratios. The three ratios exhibit an general trend of an overall increase with an increase of dose with the exception of 40 the largest exposure dose of $100 \mathrm{mg} / \mathrm{ml}$ which has been shown to have a saturated spectral/exposure response (Figure 3). All spectral features, with the exception of the $1338 \mathrm{~cm}^{-1}$ band are far from any SWCNT bands, and the fact that the $1338 \mathrm{~cm}^{-1}$ band exhibits the same trends indicates that there is minimal 45 interference from the underlying tail of the SWCNT D-line. Figure 9 shows an approximately linear relationship between the ratio of $1338 \mathrm{~cm}^{-1} /$ amide III as a function of G-line intensity which should more accurately represent the actual SWCNT dose.

The ratios of bands previously identified as cytotoxic markers 50 clearly show a dose dependent response. This dose dependence correlates well with that previously observed for colony size in clonogenic assays on the same samples ${ }^{12}$. The dose dependent response of the colony size endpoint of the clonogenic study is plotted in (Figure 10). A monotonic decrease in colony size with 55 increasing dose up to $\sim 30 \mathrm{mg} / \mathrm{l}$ is observed with a saturated response at higher doses. This toxic response has been attributed to a reduced proliferative capacity as a result of medium depletion caused by adsorption of components of the cell growth medium to the SWCNTs ${ }^{12}$. Figure 11 demonstrates a clear 60 correlation of the dose dependent $1287 \mathrm{~cm}^{-1} /$ amide III peak ratio with toxic response as determined by the colony size endpoint of reference $^{12}$.

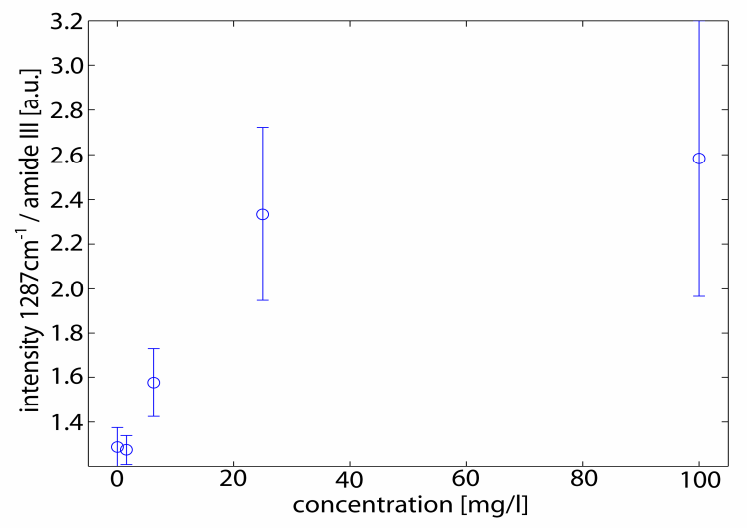

65

Figure 6. Peak ratio of $1287 \mathrm{~cm}^{-1} /$ amide III versus concentration 


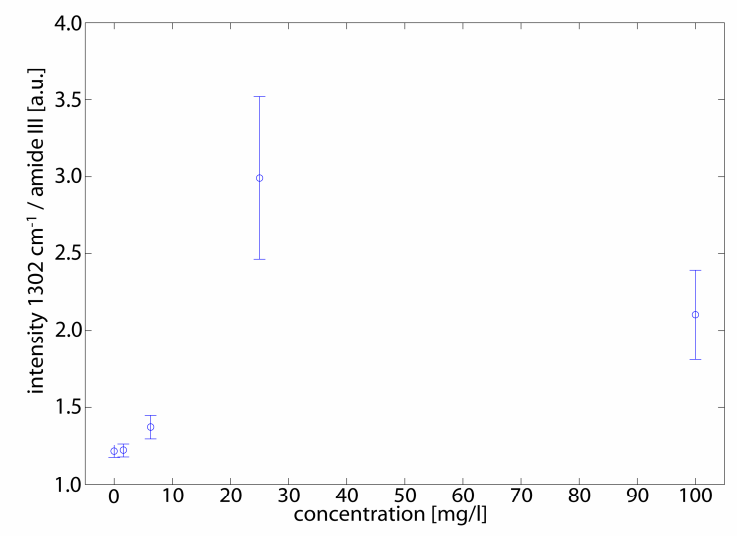

Figure 7. Peak ratio of $1302 \mathrm{~cm}^{-1} /$ amide III versus concentration

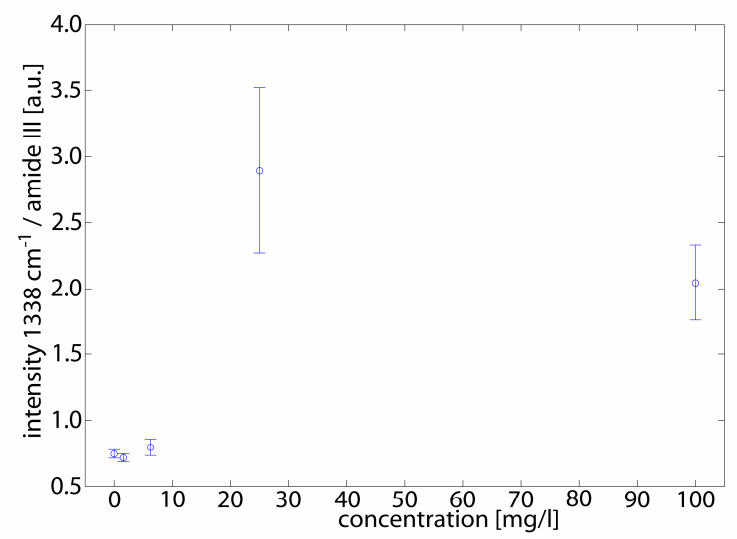

Figure 8. Peak ratio of $1338 \mathrm{~cm}^{-1} /$ amide III versus concentration

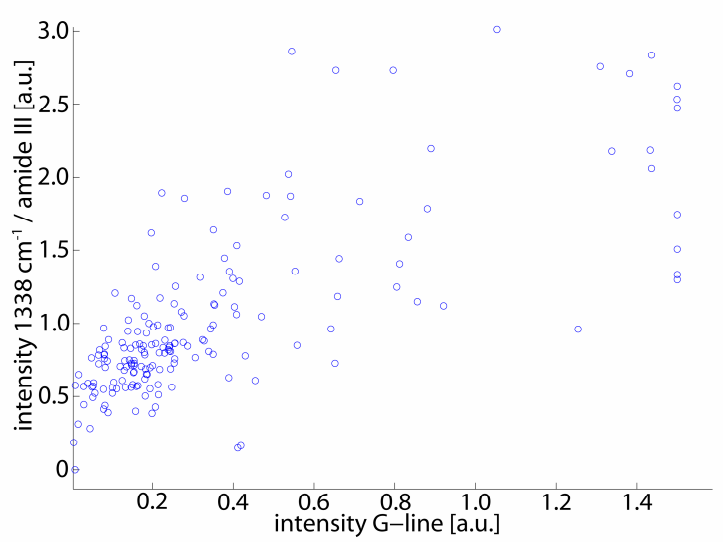

Figure 9. Peak ratio1338 $\mathrm{cm}^{-1} /$ amide III / G-Line

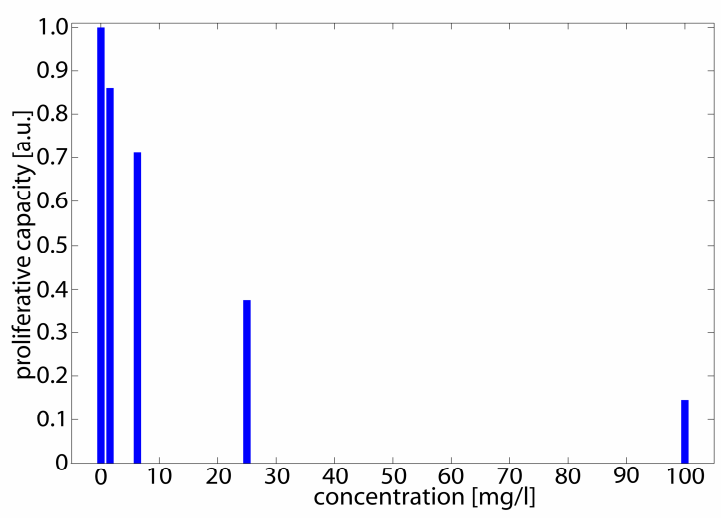

Figure 10. Dose dependent colony size of A549 cells derived by clonogenic assays (Herzog et al. ToxLett. 2007)

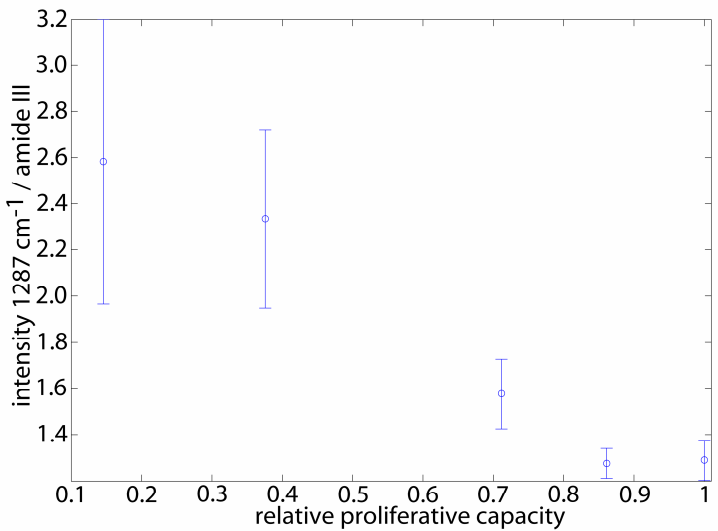

Figure 11. Correlation of the $1287 \mathrm{~cm}^{-1} /$ amide III peak ratio with colony size endpoint.

15 The results clearly indicate that dose dependent spectral markers can be identified in the Raman spectra of cellular samples exposed to SWCNTs. However, the intrinsic influences of inhomogenity of the spatial dispersion of SWCNTs in e.g. cell culture medium $^{11,46}$ and the SWCNT residues adhering to the 20 cells, as well as the complex changes to the spectral response of the cells, demand more elaborate data analysis methods, moving from the univariate approaches described above to the analysis of the spectral data by multivariate analysis. Principle component analysis will thus be employed as a more powerful classification 25 tool, potentially elucidating a more detailed signature of the cellular response.

\section{Multivariate Analysis}

The loadings from the PCA of the un-derivatized data (Figure 12) are used to monitor the spectral features according to their 30 contribution to the variance in the dataset. The highest variance, describing $68.2 \%$ of the overall variance, is represented by $\mathrm{PC}_{1}$ which is dominated by the strong features of the control variable, SWCNTs, as expected. The largest variances related to biological response due to exposure with SWCNTs, are expressed by $\mathrm{PC}_{2}$ 35 and $\mathrm{PC}_{3}$ although they represent only a further $25 \%$ variance. Within the first five components, compared to the control cell spectrum (Figure 2), component three shows the most similar features, indicating a defined response at $\sim 1030, \sim 1300, \sim 1450$ $\mathrm{cm}^{-1}$ implying a change of spectral variance due to activity in 
lipid related bands ${ }^{39}$ corroborating the peak ratio analysis of Figure 6-8. In detail, changes in the region $1230 \mathrm{~cm}^{-1}$ to $1350 \mathrm{~cm}^{-1}$ associated with the Amide III band at $1238 \mathrm{~cm}^{-1}$, DNA bases guanine, adenine and thymine at $1287 \mathrm{~cm}^{-1}$ and $1338 \mathrm{~cm}^{-1}$, and 5 lipid deformation modes at $1302 \mathrm{~cm}^{-1}$, feature strongly positive in $\mathrm{PC}_{3}$ and less strong in $\mathrm{PC}_{5}$, although multiple features of loadings of a variable in different PCs are complex to interpret.
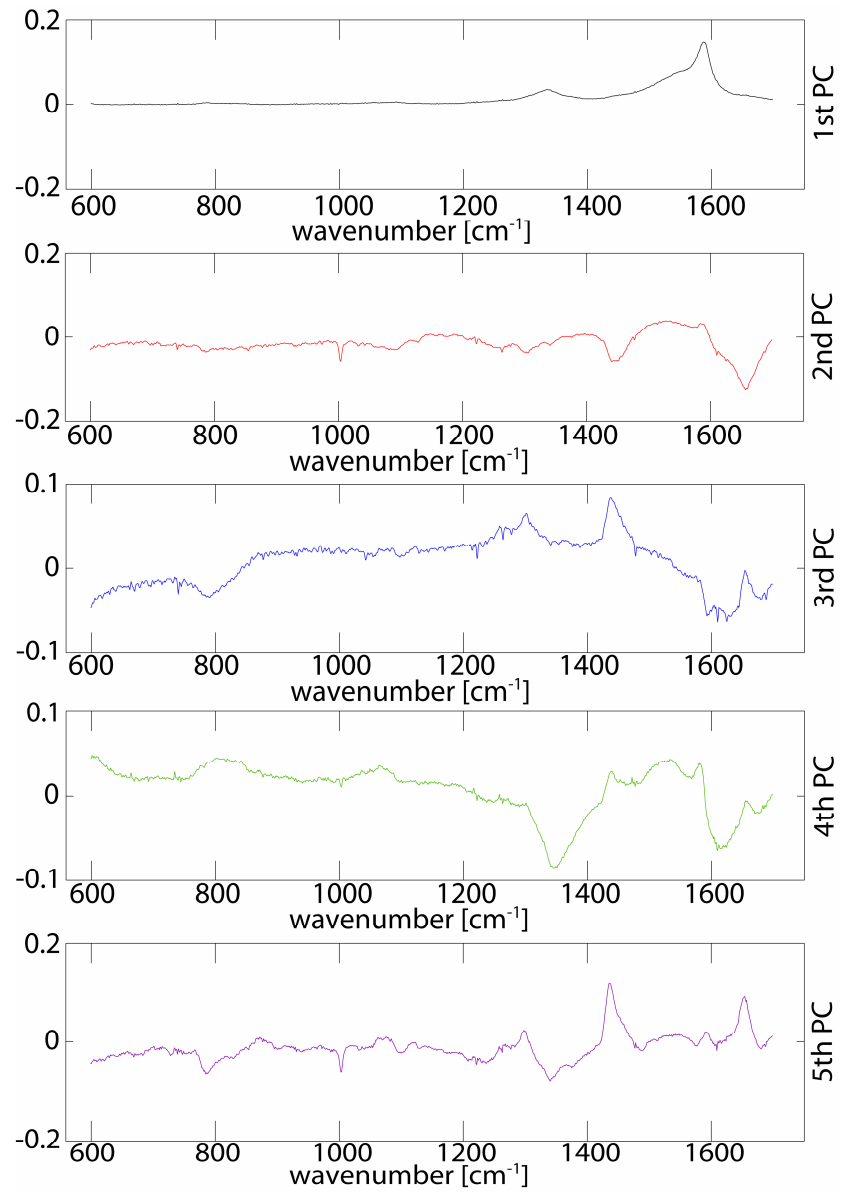

Figure 12. Individual principal component loadings plot of the first 5 10 components, (PC1-PC5 with explained variance of $68.2 \%, 20.3 \%, 4.5 \%$, $2.5 \%, 1.6 \%)$

The scores plot of the PCA shows a degree of separation into two classes between exposed and unexposed populations (Figure 13) in the $2 \mathrm{D}$ vector space spun by $\mathrm{PC}_{2}$ and $\mathrm{PC}_{3}$. However, many of 15 the exposed populations group with the unexposed populations, indicative of a non-uniform exposure. It is clear from visual observation at high concentrations ${ }^{9}$ and the variability of the contribution of the G-line as shown in figure 9, that the spatial distribution and thus local concentration of the SWCNTs varies 20 considerably from point to point in the sample at each dose. The separation or distinction between the five different exposure doses is not therefore very clear and a continuous variation of dosages as measured using the high spatial resolution of the laser is inferred. By doubly derivatizing the data, the scores plot of the 25 PCA shows distinct separations down to the level of exposure concentration of the samples, giving a defined cellular response and spatially denser co localisation of each group (Figure 14). The plot demonstrates a well defined dose dependent response

but again highlights the difficulties of establishing completely 30 homogeneous exposure doses.

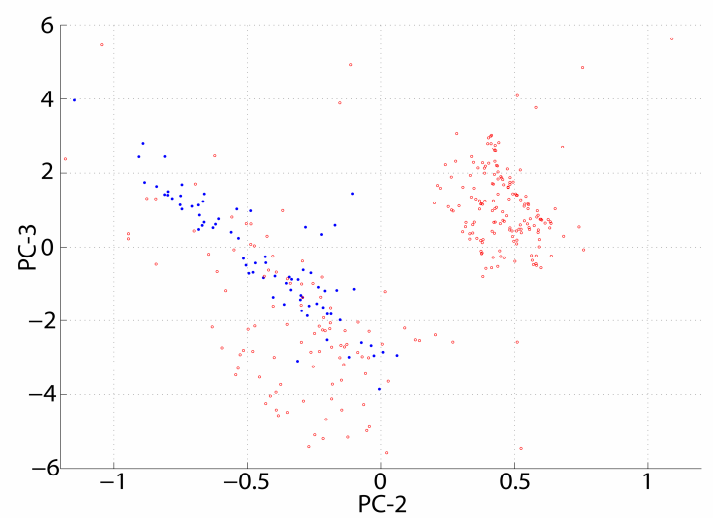

Figure 13. Principal Component Score Plot of PC2-PC3 for every exposed (red) and control population (blue)

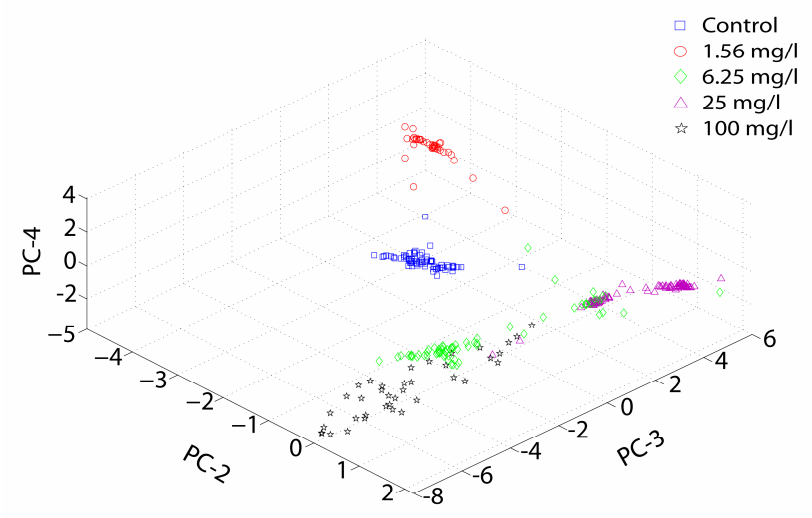

35 Figure 14. Principal Component Score Plot of PC2-PC4 for every spectrum of exposed concentration (control, 1.56, 6.25, 25.0, $100.0 \mathrm{mg} / \mathrm{l}$ ) after double derivatization

40

\section{Partial Least Squares Analysis}

This study uses the same cell populations described in Herzog et $\mathrm{al}^{12}$ and the cells under investigation here were identical in exposure to those used in the previous work. Therefore these cells 45 should reach identical clonogenic endpoints. The results of the clonogenic study are given in (Figure 10). These targets have therefore been used in a PLS model with the aim of demonstrating that the technique can potentially be extended to the determination of toxicity through the presentation of spectra 50 of samples of unknown dosage. It is hoped that this work will have advantages in the analysis of the toxic response of SWCNTs, and indeed other nanoparticles.

In total 130 Raman spectra, $60 \%$ (192 spectra) of the full set formed the training data for construction of the PLS model 55 described in this work. A GA was applied to reduce the number of wavenumbers required for prediction. Table 1 gives details of the Genetic algorithm parameters. 
Table 2: Genetic algorithm parameters

$\begin{array}{cc}\text { Parameter } & \text { Value } \\ \text { Chromosome size } & 30 \\ \text { Max. genes per chromosome } & 30 \\ \text { Mutation probability } & 0.01 \\ \text { Crossover probability } & 0.5 \\ \text { Pre-processing } & \text { None } \\ \text { Max LV } & 15 \\ \text { \#runs } & 100\end{array}$

The aim of the genetic algorithm was to minimize the RMSECV 5 for the calibration model in predicting the clonogenic endpoints of CNT induced toxicity. The GA was performed over 100 runs; the fittest individuals used 178 variables, reducing the original dataset by 559 wavenumbers. In order to choose the optimum number of LVs to be retained, rigorous cross validation was 10 carried out on the calibration set. (Figure 15) shows the results of the cross validation. Ten LVs were retained for model construction as the RMSECV did not decrease significantly after this point.

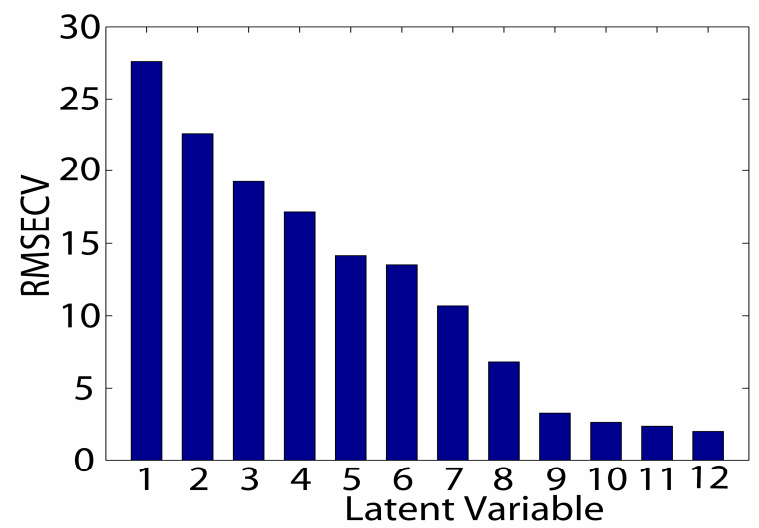

15 Figure 15. Cross validation results. The lowest RMSECV was observed at 10 latent variables $(\mathrm{RMSECV}=2.53)$.

The RMSECV was calculated to be 2.10. Table 2 shows the performance of the GA PLS.

Table 3: Performance of PLS and GA optimized PLS. The RMSE for the 20 test set (129 spectra) and training set is shown. 10 latent variables were retained for each model.

$\begin{array}{lccccc} & \text { \#wavenumbers } & \begin{array}{c}\text { LVs } \\ \text { retained }\end{array} & \text { RMSECV } & \text { RMSEC } & \text { RMSEP } \\ \text { PLS } & 178 & 10 & 4.31 & 3.17 & \begin{array}{c}3.37(\mathrm{R} 2 \\ =0.99)\end{array} \\ \text { GA- } & 149 & 10 & 2.53 & 2.10 & \begin{array}{c}2.78\left(\mathrm{R}^{2}\right. \\ =0.99)\end{array} \\ P L S & & & & & \end{array}$

Using 10 latent variables of the GA, PLS clearly outperforms multivariate calibration using the entire wavelength range, 25 showing a decrease in all RMSE values. The independent testing set held back from training was used to determine whether over fitting had occurred. Upon presentation of the testing set, the RMSEP was calculated to be 2.78 indicating an accurate model, and furthermore no over fitting was observed. Figure 16 shows a 30 plot of the predicted toxicity based on the spectral variations of the test set versus the cellular toxicity as measured in the clonogenic study ${ }^{12}$. Therefore, an accurate GA optimised PLS model has been created correlating Raman spectra to clonogenic endpoints thereby potentially reducing toxicity analysis time and 35 the cost of analysis by negating the need for post exposure cell culture. While the RMSE values observed in this study are encouraging, a further reduction in these values would be beneficial. Work is also ongoing toward the inclusion of various exposure time points, greater range of concentrations and the 40 evaluation of Raman spectroscopy for the determination of cellular toxicity mediated by other types of nanomaterials.

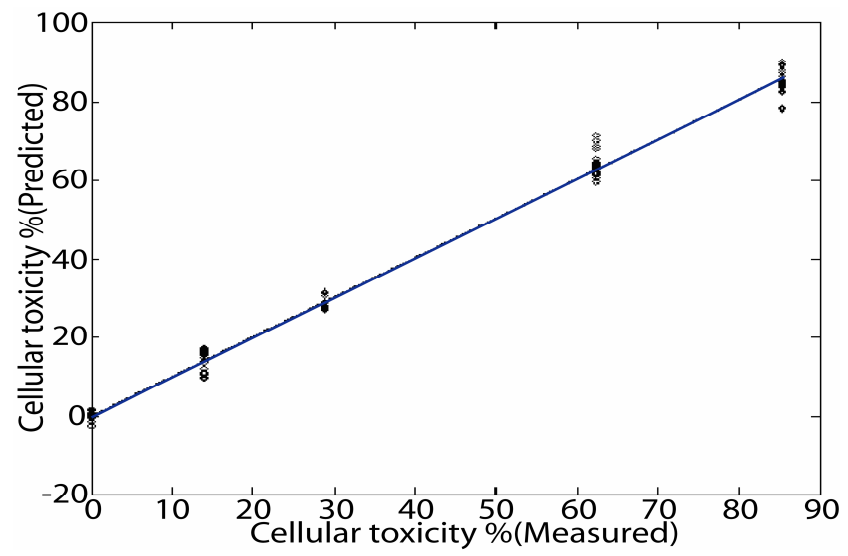

Figure 16. GA optimised PLS model correlating Raman spectra to clonogenic endpoints

${ }_{45}$ Raman spectroscopy can thus be considered as a potential technique for monitoring SWCNT induced biochemical changes at the cellular level. It is hoped that this work will have advantages in the analysis of the toxic response of SWCNTs, and indeed other nanoparticles.

\section{${ }_{50}$ Conclusions}

Although the data shown are preliminary, the potential of Raman spectroscopy as a viable tool to assess toxicology is demonstrated. A good correlation is seen between previously identified spectral markers of toxicity and the exposure dose. The ${ }_{55}$ study uses the same cell populations described in Herzog et $\mathrm{al}^{12}$ and the cells under investigation here were identical in exposure to those used in the previous work. A similarly good correlation between the spectral markers and the clonogenic endpoint of proliferative capacity is observed, indicating that the technique ${ }_{60}$ can potentially overcome the previously identified problems with colorometric assays in determining the cytotoxicity of carbon nanotubes. The changes in the spectra are visibly observable, dose dependent and associated with cytological data throughout, emphasizing that Raman spectroscopy is a precise analytical 65 method for the examination of chemical and biological properties of cells.

Although the multivariate statistical approaches may appear complex and user unfriendly, they are becoming standard tools of biospectroscopy and increasingly user friendly packages are 70 becoming available. Principal Component Analysis as such shows good dose dependent separation of spectra. Raman spectroscopy and multivariate calibration via GA optimised PLS for prognosis of cellular toxicity and proliferation shows much promise. The use of GA for variable selection increases the 75 accuracy of the PLS model and reduces the number of factors required. Accurate quantitative prediction of the endpoints of clonogenic assays was possible using Raman spectroscopy and 
GA PLS. It is hoped that this work will lead to rapid Raman based methods for the determination of SWCNT toxicity. Further experiments are planned to corroborate these analyses and to conquer the limitations of the model as a result of dispersion 5 inhomogenity within the SWCNT suspensions.

\section{References}

1. A. P. Dowling, R. Clift, N. Grobert, D. Hutton, R. Oliver, O. O'Neill, J. Pethica, N. Pidgeon, J. Porritt, J. Ryan, A. Seaton, S. Tendler, M. Welland and R. Whatmore, in The Royal Society, 2004, p. 11.

10 2. S. Iijima, Nature, $1991,354,56-58$.

3. A. P. Dowling, Nanotoday, 2004, 6

4. C.-W. Lam, J. T. James, R. McCluskey and R. L. Hunter, Toxicological Sciences, 2004, 77, 126-134.

5. D. B. Warheit, T. R. Webb, C. M. Sayes, V. L. Colvin and K. L.

15 Reed, Toxicol Sci, 2006, 91, 227-236.

6. D. B. Warheit, T. R. Webb, V. L. Colvin, K. L. Reed and C. M. Sayes, Toxicological Sciences, 2007, 95, 270-280.

7. H. Yehia, R. Draper, C. Mikoryak, E. Walker, P. Bajaj, I. Musselman, M. Daigrepont, G. Dieckmann and P. Pantano, 2007, p. 208 .

8. S. Kang, M. S. Mauter and M. Elimelech, Environ Sci Technol, 2008, 42, 7528-7534.

9. M. Davoren, E. Herzog, A. Casey, B. Cottineau, G. Chambers, H. J. Byrne and F. M. Lyng, Toxicol In Vitro, 2007, 21, 438-448.

25 10. A. Casey, E. Herzog, M. Davoren, F. M. Lyng, H. J. Byrne and G. Chambers, Carbon, 2007, 45, 1425-1432.

11. A. Casey, M. Davoren, E. Herzog, F. M. Lyng, H. J. Byrne and G. Chambers, Carbon, 2007, 45, 34-40.

12. E. Herzog, A. Casey, F. M. Lyng, G. Chambers, H. J. Byrne and M. Davoren, Toxicology Letters, 2007, 174, 49-60.

13. A. Casey, E. Herzog, F. M. Lyng, H. J. Byrne, G. Chambers and M. Davoren, Toxicology Letters, 2008, 179, 78-84.

14. S. G. Chou, H. B. Ribeiro, E. B. Barros, A. P. Santos, D. Nezich, G. G. Samsonidze, C. Fantini, M. A. Pimenta, A. Jorio, F. Plentz, M. S.

35 Dresselhaus, G. Dresselhaus, R. Saito, M. Zheng, G. B. Onoa, E. D. Semke, A. K. Swan, M. S. Unlu and B. B. Goldberg, Chemical Physics Letters, 2004, 397, 296-301.

15. G. Perna, M. Lastella, M. Lasalvia, E. Mezzenga and V. Capozzi, Journal of Molecular Structure, 2007, 834, 182-187.

40 16. F. M. Lyng, E. O. Faolain, J. Conroy, A. D. Meade, P. Knief, B. Duffy, M. B. Hunter, J. M. Byrne, P. Kelehan and H. J. Byrne, Exp Mol Pathol, 2007, 82, 121-129.

17. A. D. Meade, F. M. Lyng, P. Knief and H. J. Byrne, Analytical and Bioanalytical Chemistry, 2007, 387, 1717-1728.

45 18. I. Notingher, S. Verrier, S. Haque, J. M. Polak and L. L. Hench, Biopolymers, 2003, 72, 230-240.

19. M. Scholz, S. Gatzek, A. Sterling, O. Fiehn and J. Selbig, Bioinformatics, 2004, 20, 2447-2454.

20. C. A. Owen, J. Selvakumaran, I. Notingher, G. Jell, L. L. Hench and M. M. Stevens, J Cell Biochem, 2006, 99, 178-186.

21. T. Hasegawa, J. Nishijo and J. Umemura, Chemical Physics Letters, 2000, 317, 642-646.

22. K. J. Frost and R. L. McCreery, Applied Spectroscopy, 1998, 52, 1614-1618.

55 23. J. Ariai and S. R. P. Smith, Journal of Physics C-Solid State Physics, 1981, 14, 1193-1202.

24. A. Savitsky and M. J. E. Golay, Analytical Chemistry, 1964, 36, 1627-1639.

25. C. Chatfield and A. J. Collins, Introduction to multivariate Analysis, 60 Chapman \& Hall, London, 1980.

26. L. L. Harlow, The Essence of Multivariate Thinking - Basic Themes and Methods, Routledge, 2005.

27. G. Downey, P. McIntyre and A. N. Davies, Applied Spectroscopy, 2003, 57, 158-163.

65 28. D. M. Zhang and D. Ben-Amotz, Applied Spectroscopy, 2000, 54, 1379-1383.

29. D. M. Haaland and E. V. Thomas, Analytical Chemistry, 1988, 60, 1193-1202.
30. H. a. N. Martens, T, Multivariate Calibration, Wiley, 1989.

70 31. S. Wold, H. Martens and H. Wold, Lecture Notes in Mathematics, 1983, 973, 286-293.

32. R. Leardi, Journal of Chemometrics, 2000, 14, 643-655.

33. R. Leardi and A. L. Gonzalez, Chemometrics and Intelligent Laboratory Systems, 1998, 41, 195-207.

75 34. D. Jouanrimbaud, D. L. Massart, R. Leardi and O. E. Denoord, Analytical Chemistry, 1995, 67, 4295-4301.

35. M. S. Dresselhaus, A. Jorio, A. G. Souza, G. Dresselhaus and R. Saito, Physica B-Condensed Matter, 2002, 323, 15-20.

36. H. Kuzmany, B. Burger, M. Hulman, J. Kurti, A. G. Rinzler and R. E. Smalley, Europhysics Letters, 1998, 44, 518-524.

37. A. M. Keszler, L. Nemes, S. R. Ahmad and X. Fang, Journal of Optoelectronics and Advanced Materials, 2004, 6, 1269-1274.

38. S. M. Bose, S. Gayen and S. N. Behera, Physical Review B, 2005 , 72.

85 39. G. Socrates, Infrared and Raman Characteristic Group Frequencies Tables and Charts, John Wiley \& Sons, 2004.

40. N. Stone, C. Kendall, N. Shepherd, P. Crow and H. Barr, Journal of Raman Spectroscopy, 2002, 33, 564-573.

41. N. Stone, P. Stavroulaki, C. Kendall, M. Birchall and H. Barr, 90 Laryngoscope, 2000, 110, 1756-1763.

42. G. J. Puppels, H. S. P. Garritsen, G. M. J. Segersnolten, F. F. M. Demul and J. Greve, Biophysical Journal, 1991, 60, 1046-1056.

43. I. Notingher, I. Bisson, A. E. Bishop, W. L. Randle, J. M. Polak and L. L. Hench, Anal Chem, 2004, 76, 3185-3193.

95 44. T. Ida, M. Ando and H. Toraya, Journal of Applied Crystallography, 2000, 33, 1311-1316.

45. Lu L., Ward M. and M. A., Iron Steel Inst Jpn, 2003, 43, 1940-1946.

46. S. Giordani, S. D. Bergin, V. Nicolosi, S. Lebedkin, M. M. Kappes, W. J. Blau and J. N. Coleman, J Phys Chem B, 2006, 110, 1570815718. 